\title{
Gravitational trapping potential with arbitrary extra dimensions
}

\author{
Douglas Singleton* \\ Centre of Gravitation and Fundamental Metrology, \\ VNIIMS, 3-1 M. Ulyanovoy St., Moscow 119313, Russia; \\ Physics Dept., CSU Fresno, 2345 East San Ramon Ave. M/S 37 Fresno, CA 93740-8031, USA
}

(Dated: October 13, 2018)

\begin{abstract}
We extend a recently discovered, non-singular 6 dimensional brane, solution to $D=4+n$ dimensions. As with the previous 6D solution the present solution provides a gravitational trapping mechanism for fields of spin $0, \frac{1}{2}, 1$ and 2 . There is an important distinction between 2 extra dimensions and $n$ extra dimensions that makes this more than a trivial extension. In contrast to gravity in $n>2$ dimensions, gravity in $n=2$ dimensions is conformally flat. The stress-energy tensor required by this solution has reasonable physically properties, and for $n=2$ and $n=3$ can be made to asymptotically go to zero as one moves away from the brane.
\end{abstract}

\section{INTRODUCTION}

The brane world models introduced in [1, 2] have provided a new framework for addressing various questions in particle physics, such as the hierarchy problem, using non-compact extra dimensions (see [3, 4, 5, 6, 7, 8] for earlier work on non-compact extra dimensions and on solutions in extra dimensions). In these models one must give a mechanism for trapping or localizing fields with various spins $\left(0, \frac{1}{2}, 1,2\right)$ on the 4 dimensional brane of the observable Universe. The condition for determining whether a multi-dimensional field is localized or not is if the integral of its Lagrangian over the extra coordinates is finite. In the original 5 dimensional models it was found that it was not possible to gravitational trap all types of fields with the same warp factor. Spin 0 and spin 2 fields were trapped on the brane with a decreasing, exponential, warp factor, while spin $\frac{1}{2}$ fields were trapped with an increasing warp factor [2, 9]. Spin 1 fields were not localized at all [10]. In the 6 dimensional case with two extra dimensions slightly better trapping behavior was found [1]: 0,1 and 2 fields were localized with a decreasing, exponential warp factor but spin $\frac{1}{2}$ fields were again localized with an increasing, exponential warp factor. In 12 it was shown that one could localize the zero modes of all these fields with an increasing, non-exponential warp factor in 6 dimensions with one extra time-like dimension. In 13, 14] it was shown that all these fields could be localized in 6 dimensions with both extra dimensions being space-like. In particular [13, 15] gave an exact, non-singular, non-exponential trapping solution. Given the improving localization behavior with increasing spacetime dimension the natural inclination would be to look for trapping solutions with still more extra dimensions. However, it was pointed out in 16] that there is an important distinction in going from $n=2$ to $n>2$ extra dimensions. Gravity in 2 dimensions is conformally flat. For spacetime with greater than 2 dimensions vacuum spacetime can have a non-zero Weyl tensor. The present work shows that it is possible to extend (with some restrictions) the non-singular, trapping solution of 13, 15 to $D=4+n$ dimensions. (Earlier work on the localization of gravity in $n \geq 2$ extra dimensions with higher curvature terms can be found in [17]). The solution localizes all types of spin fields with a non-exponential, warp factor. The draw back of the solution is that the stress-energy tensor is selected or tuned so as to give these good features of the solutions. However the stress-energy tensor is physically reasonable in that its magnitude is peaked on the brane and decreases as one moves away from the brane. It will be shown that for the $n=2$ and $n=3$ cases it is possible to have the stress-energy tensor go to zero as one moves away from the brane. For $n \geq 4$ one can have part, but not all of the stress-energy tensor asymptotically go to zero.

It is possible to localize all spin fields in the $5 \mathrm{D}$ and $6 \mathrm{D}$ models with exponential warp factors if one introduces non-gravitational interactions. However, for reasons of simplicity, and since gravity couples universally to all types of matter, it is preferable to find a purely gravitational trapping mechanism. The solution presented here does give such a pure gravitational trapping mechanism for all types of spin fields. The warp factor is non-exponential in contrast to the work in [1, 2, 18, 19]

*Electronic address: dougs@csufresno.edu 


\section{D-DIMENSIONAL EINSTEIN EQUATIONS}

The system we consider is $D=4+n$ dimensional gravity with a cosmological constant $\Lambda$, and some matter fields. The action is

$$
S=\int d^{D} x \sqrt{-{ }^{D} g}\left[\frac{M^{n+2}}{2}\left({ }^{D} R+2 \Lambda\right)+L_{m}\right]
$$

where $\sqrt{-^{D} g}$ is the determinant, $M$ is the fundamental mass scale, ${ }^{D} R$ is the scalar curvature, $\Lambda$ is the cosmological constant and $L$ is the Lagrangian of matter fields. All of these quantities are $D=4+n$ dimensional. The D-dimensional Einstein equations resulting from this action are

$$
{ }^{D} R_{A B}-\frac{1}{2} g_{A B}{ }^{D} R=\Lambda g_{A B}+\frac{1}{M^{n+2}} T_{A B}
$$

Capital Latin indices run over $A, B, \ldots=0,1,2,3, \ldots, D$. The stress-energy tensor, $T_{A B}$, is determined from the matter Lagrangian, $L_{m}$, in the standard way.

The ansatz we will use is a simple extension of refs. [13, 14] with two scale functions - one for the 4-dimensional brane and one for the $n$ extra spatial dimensions.

$$
d s^{2}=\phi^{2}(r) \eta_{\alpha \beta}\left(x^{\nu}\right) d x^{\alpha} d x^{\beta}-\lambda(r)\left(d r^{2}+r^{2} d \Omega_{n-1}^{2}\right)
$$

The metric of ordinary 4-dimensional spacetime, $\eta_{\alpha \beta}\left(x^{\nu}\right)$, has the signature $(+,-,-,-)$. The Greek indices $\alpha, \beta, \ldots=$ $0,1,2,3$ refer to the coordinates of these 4-dimensions. It is assumed that ansatz (3) only depends on the extra coordinate $r$ through the 4 -dimensional conformal factors, $\phi^{2}$, and $\lambda . d \Omega_{n-1}^{2}$ is the solid angle for the $(n-1)$ sphere and is explicitly given by

$$
d \Omega_{n-1}^{2}=d \theta_{n-1}^{2}+\sin ^{2} \theta_{n-1} d \theta_{n-2}^{2}+\sin ^{2} \theta_{n-1} \sin ^{2} \theta_{n-2} d \theta_{n-3}^{2}+\cdots+\prod_{i=2}^{n-1} \sin ^{2} \theta_{i} d \theta_{1}^{2}
$$

The angles have the following ranges: $0 \leq \theta_{1} \leq 2 \pi$ and $0 \leq \theta_{i} \leq \pi$ for $i=2, \ldots, n-1$. The radial coordinate has the range $0 \leq r<\infty$.

The above generalization of the 6D ansatz of [13, 14] has an important difference from the generalization of the 6D metric ansatz considered in [11, 18, 19]. In these latter papers the scale factor, $\lambda(r)$, multiples only the angular part of the metric

$$
d s^{2}=\phi^{2}(r) \eta_{\alpha \beta}\left(x^{\nu}\right) d x^{\alpha} d x^{\beta}-d r^{2}-\lambda(r) d \theta^{2}
$$

whereas in 13, 14] the scale factor multiplies the entire extra dimensional part. Both the 6D version of (3) and (5) are locally the same. This can be seen by defining $d r$ of (3) via $\lambda(r) d r^{2}=d \tilde{r}^{2}$ where $\tilde{r}$ is the radial coordinate of (5). However there is a global distinction between (3) and (5). The metric in (5) corresponds to a cone-like geometry such as occurs with string-like defect. For example, if in (5) one has $\lambda(r)=(1-n)^{2} r^{2}$ then the geometry of the extra space will exhibit an angular deficit of $2 \pi n$. A similar $\lambda(r)$ in (3) would not result in an angular deficit.

For the 6D ansatz of [13, 14] and its generalization to $4+\mathrm{n}$ given in (3) the metric functions, $\lambda(r)$ and $\phi(r)$, are conformal factors for an n-dimensional Euclidean metric and 4-dimensional Minkowski metric respectively. Thus as long as the metric functions are well behaved (i.e. not equal to 0 or $\infty$ ) the metric will be non-singular. Since we are looking for non-singular solutions, we choose the metric to take the form (3).

The nonzero components of the stress-energy tensor $T_{A B}$ are taken to be of the form

$$
T_{\mu \nu}=-g_{\mu \nu} F(r), \quad T_{i j}=-g_{i j} K(r), \quad T_{i \mu}=0
$$

The two source functions, $F(r)$ and $K(r)$, depend only on the radial coordinate $r$.

As a final simplification we assume that 4-dimensional metric is flat $\left(\eta_{\alpha \beta}\left(x^{\nu}\right)=\eta_{\alpha \beta}\right)$ and that the 4-dimensional cosmological constant is zero. This yields

$$
{ }^{4} R_{\mu \nu}-\frac{1}{2} \eta_{\mu \nu}{ }^{4} R=0
$$


where ${ }^{4} R_{\alpha \beta},{ }^{4} R$ are the 4 -dimensional Ricci tensor and scalar constructed from $\eta_{\alpha \beta}$. With these simplifications and the ansätze of (3) and (6) the D-dimensional Einstein field equations (2) become [20]

$$
\begin{aligned}
& 3\left(2 \frac{\phi^{\prime \prime}}{\phi}-\frac{\phi^{\prime}}{\phi} \frac{\lambda^{\prime}}{\lambda}\right)+6 \frac{\left(\phi^{\prime}\right)^{2}}{\phi^{2}}+(n-1)\left[3 \frac{\phi^{\prime}}{\phi} \frac{\left(r^{2} \lambda\right)^{\prime}}{r^{2} \lambda}+\frac{\left(r^{2} \lambda\right)^{\prime \prime}}{r^{2} \lambda}+\frac{n-4}{4}\left(\frac{\left(r^{2} \lambda\right)^{\prime}}{r^{2} \lambda}\right)^{2}-\frac{1}{2} \frac{\lambda^{\prime}}{\lambda} \frac{\left(r^{2} \lambda\right)^{\prime}}{r^{2} \lambda}-\frac{n-2}{r^{2}}\right] \\
& =2 \lambda\left(\frac{F(r)}{M^{n+2}}-\Lambda\right), \\
& 12 \frac{\left(\phi^{\prime}\right)^{2}}{(\phi)^{2}}+(n-1)\left[4 \frac{\phi^{\prime}}{\phi} \frac{\left(r^{2} \lambda\right)^{\prime}}{r^{2} \lambda}+\frac{(n-2)}{4}\left(\frac{\left(r^{2} \lambda\right)^{\prime}}{r^{2} \lambda}\right)^{2}-\frac{(n-2)}{r^{2}}\right]=2 \lambda\left(\frac{K(r)}{M^{n+2}}-\Lambda\right), \\
& 4\left(2 \frac{\phi^{\prime \prime}}{\phi}-\frac{\phi^{\prime}}{\phi} \frac{\lambda^{\prime}}{\lambda}\right)+12 \frac{\left(\phi^{\prime}\right)^{2}}{\phi^{2}}+(n-2)\left[4 \frac{\phi^{\prime}}{\phi} \frac{\left(r^{2} \lambda\right)^{\prime}}{r^{2} \lambda}+\frac{\left(r^{2} \lambda\right)^{\prime \prime}}{r^{2} \lambda}+\frac{n-5}{4}\left(\frac{\left(r^{2} \lambda\right)^{\prime}}{r^{2} \lambda}\right)^{2}-\frac{1}{2} \frac{\lambda^{\prime}}{\lambda} \frac{\left(r^{2} \lambda\right)^{\prime}}{r^{2} \lambda}-\frac{n-3}{r^{2}}\right] \\
& =2 \lambda\left(\frac{K(r)}{M^{n+2}}-\Lambda\right) \text {, }
\end{aligned}
$$

where the prime $=\partial / \partial r$. These equations are for the $\alpha \alpha, r r$, and $\theta \theta$ components respectively.

Subtracting the $r r$ component of (9) from the $\theta \theta$ component of (10) to cancel the source and cosmological terms yields

$$
8\left(\frac{\phi^{\prime \prime}}{\phi}-\frac{\lambda^{\prime}}{\lambda} \frac{\phi^{\prime}}{\phi}-\frac{\phi^{\prime}}{r \phi}\right)+(n-2)\left(\frac{\lambda^{\prime \prime}}{\lambda}-\frac{3}{2}\left(\frac{\lambda^{\prime}}{\lambda}\right)^{2}-\frac{\lambda^{\prime}}{r \lambda}\right)=0 .
$$

For $n=2$ one has the simplification that the second term above does not appear. Following [20] we look for solutions in which each term in parentheses above is zero separately

$$
\frac{\phi^{\prime \prime}}{\phi}-\frac{\lambda^{\prime}}{\lambda} \frac{\phi^{\prime}}{\phi}-\frac{\phi^{\prime}}{r \phi}=0 \quad, \quad \frac{\lambda^{\prime \prime}}{\lambda}-\frac{3}{2}\left(\frac{\lambda^{\prime}}{\lambda}\right)^{2}-\frac{\lambda^{\prime}}{r \lambda}=0
$$

A solution to the first equation in (12) is

$$
\lambda(r)=\rho^{2} \frac{\phi^{\prime}(r)}{r},
$$

where $\rho$ is an integration constant with dimensions of length. In [20] it was shown that the trapping solution of [14] could be generalized to more than two extra dimensions. The following metric functions

$$
\phi(r)=a \frac{r^{2}-c^{2}}{r^{2}+c^{2}} \quad, \quad \lambda(r)=\frac{4 a c^{2} \rho^{2}}{\left(r^{2}+c^{2}\right)^{2}},
$$

solve (12), with $a$ and $c$ constants. In [14] the solution was more general in that one had $r^{2}, c^{2} \rightarrow r^{b}, c^{b}$. We will find a similar selection of the exponent when we generalize the non-singular solution of [13, 15] to $n>2$ dimensions. The solution (14) becomes singular at $r=c$ and thus was taken as a solution for $r>\epsilon$ where $\epsilon$ is the brane width, and the condition, $\epsilon \geq c$, holds. Outside $r=\epsilon$ the scale factor for 4-dimensional space, $\phi^{2}(r)$, asymptotically approaches the value $a^{2}$. If the brane width, $\epsilon$, is small then the 4 dimensional scale factor changes very quickly from $\phi^{2}(0)=1$ to $\phi^{2}(\infty)=a^{2}$. In $6 \mathrm{D}$ the solution in (14) is supported by a stress-energy tensor whose source ansatz functions, $F(r), K(r)$ where proportional to $\frac{1}{\phi^{2}}$. The solution $\phi(r)$ in (14) was shown 14, 20] to localize fields of all spins up to 2 on the brane.

In 13, 15] a solution was given which had similar properties to the solution of (14) (non-exponential warp factor which localized fields of all spins up to spin 2), but which was non-singular. Here it is shown that, with some modifications and restrictions, it is possible to extend this solution to $n$ extra spatial dimensions. This is more than a trivial exercise since for $n=2$ the extra space is conformally flat, while for $n>2$ this is not necessarily the case. Also for both $n=2$ and $n=3$ it is possible to make a choice of parameters (i.e. $\epsilon, a, \Lambda$ ) such that $F(r)$ and $K(r)$ both have the nice feature of going to zero as $r \rightarrow \infty$. For $n \geq 4$ one can make either $F(r)$ or $K(r)$ go to zero, but not both.

It is straightforward to check that the non-singular solution of [13, 15]

$$
\phi(r)=\frac{c^{2}+a r^{2}}{c^{2}+r^{2}} \quad, \quad \lambda(r)=\frac{2(a-1) c^{2} \rho^{2}}{\left(c^{2}+r^{2}\right)^{2}}
$$


satisfies both equations from (12); $a$ and $c$ are constants. The solution given in [15] was more general than (15] having the form

$$
\phi(r)=\frac{c^{b}+a r^{b}}{c^{b}+r^{b}}
$$

where the exponent of $r$ could take values other than 2 and was restricted by $|b| \geq 2$. In the present case if one inserts the $\phi(r)$ from (16) into the second equation in (12) (the first is automatically satisfied via the choice in (13)) one obtains

$$
\left(\frac{\lambda^{\prime \prime}}{\lambda}-\frac{3}{2}\left(\frac{\lambda^{\prime}}{\lambda}\right)^{2}-\frac{\lambda^{\prime}}{r \lambda}\right) \rightarrow \frac{4-b^{2}}{2 r^{2}}
$$

which only vanishes for the choice $b= \pm 2$. Thus for $n>2$ the $b= \pm 2$ solution of [15] is selected. In [15] the $b \geq 2$ solutions gave a scale factor, $\phi(r)$, which monotonically increased as one moved away from the brane. The $b \leq 2$ solutions gave a scale factor, $\phi(r)$, which decreased monotonically as one moved away from the brane. For the solution in (15) the increasing $\phi(r)$ corresponds to $a>1$ and the decreasing $\phi(r)$ to $a<1$. It was pointed out in [15] that both increasing and decreasing solutions give localization of fields with spins ranging from 0 to 2 .

Some of the parameters in (15) can be fixed. The width of the brane, $\epsilon$, is given by the inflection point of $\phi(r)$, i.e. $\phi^{\prime \prime}(r=\epsilon)=0$. This conditions gives $c=3 \epsilon^{2}$. Next we require that $\lambda(r=0)=1$, i.e. that on the brane one has a 6-dimensional Minkowski metric. This gives $\rho^{2}=\frac{3 \epsilon^{2}}{2(a-1)}$. Putting these parameters back into (15) yields

$$
\phi(r)=\frac{3 \epsilon^{2}+a r^{2}}{3 \epsilon^{2}+r^{2}} \quad, \quad \lambda(r)=\frac{9 \epsilon^{4}}{\left(3 \epsilon^{2}+r^{2}\right)^{2}},
$$

Now taking $\phi$ and $\lambda$ from the above equation and inserting them into equations (8) and (9) we obtain expressions for the source functions $F(r)$ and $K(r)$

$$
\begin{aligned}
\frac{F(r)}{M^{n+2}=\Lambda} & +\frac{-6 \epsilon^{2} n(n-3 a+2)}{\left(3 \epsilon^{2}+a r^{2}\right)^{2}}+\frac{2\left(3(n+2)+3 a^{2}(n+2)-2 a\left(n^{2}+2 n+6\right)\right) r^{2}}{\left(3 \epsilon^{2}+a r^{2}\right)^{2}} \\
& +\frac{-2 a n(-3+a(n+2)) r^{4}}{3 \epsilon^{2}\left(3 \epsilon^{2}+a r^{2}\right)^{2}} \\
\frac{K(r)}{M^{n+2}}=\Lambda & +\frac{-6 \epsilon^{2}(n-1)(n-4 a+2)}{\left(3 \epsilon^{2}+a r^{2}\right)^{2}}+\frac{4\left(2(n+2)+2 a^{2}(n+2)-a\left(n^{2}+n+10\right)\right) r^{2}}{\left(3 \epsilon^{2}+a r^{2}\right)^{2}} \\
& +\frac{-2 a(n-1)(-4+a(n+2)) r^{4}}{3 \epsilon^{2}\left(3 \epsilon^{2}+a r^{2}\right)^{2}}
\end{aligned}
$$

For $n=2$ and using the relationship $\epsilon^{2}=\frac{40 M^{4}}{3 \Lambda}$ from 13$]$ one can show that $F(r), K(r)$ reduce to the expressions in [13]. It is straightforward to check that the last equation (10) is also satisfied by $\phi(r), \lambda(r)$ from (18) and $K(r)$ from (20). One can also check that the stress-energy tensor defined by $F(r)$ and $K(r)$ above satisfy energy-momentum conservation given by

$$
\nabla^{A} T_{A B}=\frac{1}{\sqrt{-^{D} g}} \partial_{A}\left(\sqrt{-{ }^{D} g} T^{A B}\right)+\Gamma_{C D}^{B} T^{C D}=0
$$

In terms of the source ansatz functions this yields

$$
K^{\prime}+4 \frac{\phi^{\prime}}{\phi}(K-F)=0
$$

Using the $F(r)$ and $K(r)$ from (19) one finds that indeed (22) is satisfied. Thus taken together (18) (19) (20) give a non-singular, trapping solution to the $4+n$ dimensional Einstein equations (8) (91) (10).

\section{LOCALIZATION OF ZERO MODES}

As with the $6 \mathrm{D}$ solution of [13, 15] the present solution provides a gravitational trapping for fields of various spins: $0, \frac{1}{2}, 1,2$. The condition for a field to be trapped on the brane is that the integral of the Lagrangian over the extra coordinates is finite.

$$
S_{s}=\int d^{D} x \sqrt{-{ }^{D} g} L_{s}=\int d \Omega_{n-1} \int d r \phi^{4} \lambda^{n / 2} r^{n-1} \int d^{4} x L_{s}
$$


where $L_{s}$ is the matter Lagrangian for fields with spin $s=0, \frac{1}{2}, 1,2$. We will outline the trapping of the zero modes for each of these types of fields by applying the solution (18) to the analysis given in [20]. The Lagrangians for the various spin fields will contribute different factors of $\phi, \lambda$ and $r$ to the common factor of $\sqrt{-{ }^{D} g}=\phi^{4} \lambda^{n / 2} r^{n-1}$ in (23).

For a scalar field the specific from of the action is

$$
S_{0}=-\frac{1}{2} \int d^{D} x \sqrt{-{ }^{D} g} g^{M N} \partial_{M} \Phi \partial_{N} \Phi
$$

The equations of motion from this scalar Lagrangian have a zero-mode solution [20] of the form $\Phi\left(x^{M}\right)=\phi\left(x^{\mu}\right) u_{0}$, with $u_{0}$ being the constant solution for the extra dimensions, and $\phi\left(x^{\mu}\right)$ satisfies the Klein-Gordon equation on the brane $\eta^{\mu \nu} \partial_{\mu} \partial_{\nu} \phi(x)=0$. Thus the scalar action becomes

$$
S_{0}=-\frac{\pi^{\frac{n}{2}}}{\Gamma\left(\frac{n}{2}\right)} u_{0}^{2} \int_{0}^{\infty} d r \phi^{2} \lambda^{n / 2} r^{n-1} \int d^{4} x \eta^{\mu \nu} \partial_{\mu} \phi \partial_{\nu} \phi
$$

The standard gamma function above comes from the integral over $d \Omega_{n-1}$ namely $\int d \Omega_{n-1}=\frac{2 \pi^{\frac{n}{2}}}{\Gamma\left(\frac{n}{2}\right)}$. The extra factor of $\phi^{-2}$ comes from $g^{\mu \mu}$ in the Lagrangian. Inserting the solution into the radial integral we obtain

$$
\begin{aligned}
\int_{0}^{\infty} d r \phi^{2} \lambda^{n / 2} r^{n-1} & =3^{n} \epsilon^{2 n} \int_{0}^{\infty} r^{n-1} \frac{\left(3 \epsilon^{2}+a r^{2}\right)^{2}}{\left(3 \epsilon^{2}+r^{2}\right)^{n+2}} d r \\
& =\frac{3^{n / 2} \epsilon^{n}}{2^{n+1}}\left(a \sqrt{\pi} \frac{\Gamma\left(1+\frac{n}{2}\right)}{\Gamma\left(\frac{3+n}{2}\right)}+2^{n}\left(1+a^{2}\right) \frac{\Gamma\left(2+\frac{n}{2}\right) \Gamma\left(\frac{n}{2}\right)}{\Gamma(2+n)}\right)
\end{aligned}
$$

which is finite.

The action for the spinor case is

$$
S_{1 / 2}=\int d^{D} x \sqrt{-{ }^{D} g} \bar{\Psi} i \Gamma^{M} D_{M} \Psi .
$$

The curved spacetime gamma matrices $\left(\Gamma^{M}\right)$ are related to the flat spacetime gamma matrices $\left(\gamma^{M}\right)$ via the inverse vielbein $e_{\bar{M}}^{M}, \Gamma^{M}=e_{\bar{M}}^{M} \gamma^{\bar{M}}$. The vielbein is defined via $g_{M N}=e_{M}^{\bar{M}} e_{N}^{\bar{N}} \eta_{\bar{M} \bar{N}} . D_{M} \Psi=\left(\partial_{M}+\frac{1}{4} \omega_{M}^{\bar{N} \bar{P}} \gamma_{\bar{N} \bar{P}}\right) \Psi$ is the covariant derivative. The spin connection $\omega_{M}^{\bar{N}} \bar{P}$ is defined in terms of the veilbein and its derivatives in the standard way. In [20] the zero mode for the $4+n$ dimensional Dirac equation was found to be of the form $\Psi\left(x^{A}\right)=\psi\left(x^{\mu}\right) u(r) \chi\left(\theta_{i}\right)$, where $\psi\left(x^{\mu}\right)$ satisfied the massless 4 dimensional Dirac equation, $\gamma^{\mu} \partial_{\mu} \psi=0$, and $\chi\left(\theta_{i}\right)$ satisfied the angular part of the higher dimensional Dirac equation. The specific forms of $\psi$ and $\chi$ were not needed in the analysis of the trapping of the fields. The $u(r)$ part of the higher dimensional spinor was found [20] to have the form

$$
u(r)=C_{1 / 2} \phi^{-2}\left(\lambda^{\frac{1}{2}} r\right)^{-\frac{n-1}{2}}
$$

where $C_{1 / 2}$ is an integration constant. In contrast to the other fields, for the spinor field the extra space part of the wavefunction is not simply a constant. Inserting all this into the spinor action in (27) yields

$$
\begin{aligned}
S_{1 / 2} & =\int_{0}^{\infty} d r \phi^{3} \lambda^{\frac{n}{2}} r^{n-1} u^{2}(r) \int d \Omega_{n-1} \chi^{2}\left(\theta_{i}\right) \int d^{4} x \bar{\psi} i \gamma^{\mu} \partial_{\mu} \psi \\
& =\int_{0}^{\infty} d r \phi^{-1} \lambda^{\frac{1}{2}} \int d \Omega_{n-1} \chi^{2}\left(\theta_{i}\right) \int d^{4} x \bar{\psi} i \gamma^{\mu} \partial_{\mu} \psi
\end{aligned}
$$

The extra factor of $\phi$ in the first expression comes from the veilbein of the higher dimensional gamma matrix, $\Gamma^{A}$. The integral over $d \Omega_{n-1}$ is finite [20] and the integral over $r$ is

$$
\int_{0}^{\infty} d r \phi^{-1} \lambda^{\frac{1}{2}}=\int_{0}^{\infty} d r \frac{3 \epsilon^{2}}{3 \epsilon^{2}+a r^{2}}=\frac{\sqrt{3} \epsilon \pi}{2 \sqrt{a}}
$$

which is also finite. Thus the integral over the extra coordinates is finite and the fermions are localized.

For the vector, spin 1 case the action is given by

$$
S_{1}=-\frac{1}{4} \int d^{D} x \sqrt{-{ }^{D} g} g^{M N} g^{R S} F_{M R} F_{N S}
$$


where $F_{M N}=\partial_{M} A_{N}-\partial_{N} A_{M}$. There is a zero mode solution [20] of the form $A_{M}\left(x^{N}\right)=a_{\mu}\left(x^{\nu}\right) u_{0}$ with $u_{0}$ the constant solution over the $n$ extra space dimensions and $a_{\mu}$ satisfies the normal 4 dimensional vacuum Maxwell equation, $\partial^{\mu}\left(\partial_{\mu} a_{\nu}-\partial_{\nu} a_{\mu}\right)=\partial^{\mu} f_{\mu \nu}=0$. Inserting all this along with the solution of (18) into (31) gives

$$
S_{1}=-\frac{\pi^{\frac{n}{2}}}{2 \Gamma\left(\frac{n}{2}\right)} u_{0}^{2} \int_{0}^{\infty} d r \lambda^{n / 2} r^{n-1} \int d^{4} x \eta^{\mu \nu} \eta^{\lambda \sigma} f_{\mu \lambda} f_{\nu \sigma}
$$

The radial integral is evaluated as

$$
\int_{0}^{\infty} d r \lambda^{n / 2} r^{n-1}=\int_{0}^{\infty} d r \frac{3^{n} \epsilon^{2 n} r^{n-1}}{\left(3 \epsilon^{2}+r^{2}\right)^{n}}=\frac{3^{n / 2} \epsilon^{n} \sqrt{\pi} \Gamma\left(\frac{n}{2}\right)}{2^{n} \Gamma\left(\frac{1+n}{2}\right)}
$$

Since the radial integral is finite the vector fields are also localized on the brane by this solution.

The analysis for the spin- 2 is similar to the spin- 0 case and results in the same radial integral (see [13]). Thus the present solution also localizes the 4-dimensional spin-2 graviton on the brane, since the integral (26) is finite. As for the $6 \mathrm{D}$ solution, the present solution localizes spin- $0, \frac{1}{2}, 1,2$ onto the brane via gravity alone. In all cases the integral over the extra coordinates is finite independent of whether the $4 \mathrm{D}$ scale function, $\phi^{2}$, is increasing $(a>1)$ or decreasing $(a<1)$. Thus, as pointed out in 15 , both types of solutions localize the fields. This is connected with the fact that the extra space scale function, $\lambda$, is independent of $a$, and decreases to zero away from the brane in all cases.

\section{CONCLUSIONS}

The above results are in some sense expected since the scale factors, $\phi(r), \lambda(r)$, given in (18) are of the same form as in the $6 \mathrm{D}$ case in [13]. However in contrast to the general $6 \mathrm{D}$ solution found in [15], the present higher dimensional solution selects out the $b= \pm 2$ exponent for the $r^{b}$ dependence of $\phi(r)$.

The behavior of the ansatz functions, $F(r)$ and $K(r)$, depends on the various parameters, $n, \epsilon, \Lambda$ and $a$. In particular $\Lambda$ and $a$ play the major role in determining the values of $F(r)$ and $K(r)$ at $r=0$ and $r=\infty$. For a large choice of parameters which lead to $\delta$-like behavior for $F(r)$ and $K(r)$ (i.e. peaked near $r=0$ and decreasing as $r \rightarrow \infty$ ). Let us now focus on the $r \rightarrow \infty$ limit of $F(r)$ and $K(r)$. It is possible to have either $F(r)$ or $K(r)$ or both go to zero for some choices of parameters, $a, \Lambda, \epsilon$, and $n$. To see this we first write the asymptotic values of $F(r)$ and $K(r)$ from (19)

$$
\begin{aligned}
& F(r \rightarrow \infty)=\Lambda M^{n+2}+\frac{2 n M^{n+2}(3-a(n+2))}{3 a \epsilon^{2}} \\
& K(r \rightarrow \infty)=\Lambda M^{n+2}+\frac{2(n-1) M^{n+2}(4-a(n+2))}{3 a \epsilon^{2}}
\end{aligned}
$$

It is possible to make $F(\infty)=0$ by choosing

$$
a=\frac{6 n}{4 n+2 n^{2}-3 \epsilon^{2} \Lambda}
$$

and it is possible to make $K(\infty)=0$ by choosing

$$
a=\frac{8(n-1)}{2(n-1)(n+2)-3 \epsilon^{2} \Lambda}
$$

If $\Lambda=0$ in (35) and (36) then $a=\frac{3}{n+2}$ and $a=\frac{4}{n+2}$ respectively. Thus for $\Lambda=0$ one can only have $F(r)$ or $K(r)$ asymptotically go to zero with $a<1$ solutions. One must have $\Lambda>0$ if one wants $F(r)$ or $K(r)$ asymptotically to go to zero and have $a>1$. It is also possible to have both $F(r)$ and $K(r)$ go to zero at $r \rightarrow \infty$. Setting (35) equal to (36) and solving for $3 \epsilon^{2} \Lambda$ gives

$$
3 \epsilon^{2} \Lambda=\frac{2 n(n+2)(n-1)}{n-4}
$$

Inserting this into either (35) or (36) gives $a=\frac{4-n}{2+n}$ which yields $a<1$ for $n \geq 2$. For the 6D case considered in 13] the focus was on $a>1$ solutions and thus there it was not possible to have both $F(r)$ and $K(r)$ asymptotically go 
to zero. Thus there are two possibilities to have both $F(\infty)=0$ and $K(\infty)=0: n=2$ and $n=3$ which correspond

to $a=\frac{1}{2}$ and $a=\frac{1}{5}$. For $n>4$ and $a<0$ and the solution in (15) becomes singular. The $n=4$ case also has problems. First the scale function $\phi(r)$ becomes singular at $r=\infty$. Also from (37) one finds that either $\epsilon$ or $\Lambda$ or both are infinite. Finally, if one recomputes the source ansatz functions, $F(r), K(r)$ in this case one finds that they always go to $\infty$ at $r=\infty$. However an interesting result is that for $n=3$ one has a non-singular brane solution with sources that approach zero away from the brane. In [16] it was argued that for local defects (i.e. solutions where the stress-energy was strictly zero outside the brane or decreased exponentially) it might not be possible to construct non-singular solutions for $n>2$. Our $n=3$ solution evades this restrictions since the stress-energy goes to zero as a power law rather than exponentially or being strictly zero outside the brane.

In this paper we have extended the non-singular, 6 dimensional brane, world trapping solution of 13, 15. to $n>2$ extra dimensions. This is more than a simple mathematical exercise since, as pointed out in [16], there is a significant difference between $n=2$ and $n>2$ extra dimensions. As with the 6D solution, the present solution provides a universal, gravitational trapping mechanism for fields of various spins from spin 0 to spin 2 . Considering the magnitude of the stress-energy ansatz functions necessary to realize this trapping solution one finds physically reasonable properties for a range of parameters $n, \Lambda, \epsilon$ and $a$. In addition for $n=2$ and $n=3$ it is possible to have the entire stress-energy tensor go to zero at $r=\infty$. For $n \geq 4$ one can have either $F(r)$ or $K(r)$ go to zero, but not both. We have not addressed the question of the stability of this brane solution in the present paper.

\section{ACKNOWLEDGEMENT}

This work is supported by a 2004 Fulbright Scholars Grant. DS thanks Prof. Vitaly Melnikov for the invitation to work at VNIIMS and the People's Friendship University of Russia.

[1] M. Gogberashvili, Int. J. Mod. Phys., D 11, 1639 (2002); Int. J. Mod. Phys., D 11, 1635 (2002).

[2] L. Randall and R. Sundrum, Phys. Rev. Lett. 83, 3370 (1999); ibid. 83, 4690 (1999).

[3] K. Akama, in Proceedings of the Symposium on Gauge Theory and Gravitation, Nara, Japan, eds. K. Kikkawa, N. Nakanishi, and H. Nariai, Springer-Verlag, 1983, hep-th/0001113

[4] V.A. Rubakov and M.E. Shaposhnikov, Phys. Lett. B125, 136 (1983); ibid., 139 (1983)

[5] M. Visser, Phys. Lett. B159, 22 (1985)

[6] G.W. Gibbons and D.L. Wiltshire, Nucl. Phys. B287, 717 (1987)

[7] V.N. Melnikov, Cosmology and Gravitation, ed. M. Novello, Editions Frontieres, Singapore p. 147 (1994); Cosmology and Gravitation II, ed. M. Novello, Editions Frontieres, Singapore p. 465 (1996); Exact Solutions in Multidimensional Gravity and Cosmology III, CBPF-MO-03/02, Rio de Janerio, p. 297 (2002)

[8] V.D. Ivashchuk and V.N. Melnikov, Class. Quant. Grav. 18, R82-R157 (2001)

[9] B. Bajc and G. Gabadadze, Phys. Lett. B474, 282 (2000).

[10] A. Pomarol, Phys. Lett. B486, 153 (2000).

[11] I. Oda, Phys. Rev. D 62, 126009 (2000).

[12] M. Gogberashvili and P. Midodashvili, Phys. Lett. B 515, 447 (2001); Europhys. Lett., 61, 308 (2003).

[13] M. Gogberashvili and D. Singleton, Phys. Lett. B 582, 95 (2004)

[14] M. Gogberashvili and D. Singleton, Phys. Rev. D 69, 026004 (2004)

[15] P. Midodashvili, hep-th/0308051

[16] T. Gherghetta, E. Roessl, M. Shaposhnikov, Phys. Lett. B491, 353 (2000)

[17] I. Neupane, JHEP 0009, 040 (2000); Class.Quant.Grav. 19, 5507 (2002)

[18] T. Gherghetta and M. Shaposhnikov, Phys. Rev. Lett. 85, 240 (2000).

[19] R. Gregory, Phys. Rev. Lett. 84, 2564 (2000).

[20] I. Oda, Phys. Lett. B 571, 235 (2003) 